\title{
¿ALIENADOS, REBELDES, O REVOLUCIONARIOS? EL COMUNISMO ITALIANO Y EL DESAFÍO DE LAS CULTURAS JUVENILES, 1960-1966
}

\author{
¿ALIENATED, REBELS, OR REVOLUTIONARIES? ITALIAN \\ COMMUNISM AND THE CHALLENGE OF YOUTH \\ CULTURES, 1960-1966
}

\author{
Mauro Pasqualini \\ CONICET/CIS-IDES
}

Entregado el 19-9-2013 y aceptado el 22-1-2014.

\begin{abstract}
Resumen: El artículo estudia las reacciones y debates sobre la «cuestión juvenil» en el seno de Vie Nuove, un semanario ligado al Partido Comunista Italiano durante los años '60s. Considera que las reacciones de los editores cambiaron en el lapso de pocos años. Mientras que en un principio la continuidad generacional era sinónimo de virtud política, posteriormente se comenzó a discutir acerca del valor de actitudes rupturistas por parte de los jóvenes. El artículo enfatiza la heterogeneidad de las posiciones al interior de este grupo de comunistas italianos, y su interés pero también dificultad para entender las nuevas culturas juveniles.
\end{abstract}

Palabras clave: Culturas Juveniles, 1960s, Partido Comunista Italiano, Historia de Italia.

\begin{abstract}
The article studies the responses to the «youth question» within Vie Nuove, a weekly linked to the Italian Communist Party during the 1960s. It shows that the editors' reactions changed within a short period of time. While
\end{abstract}

${ }^{1}$ El autor desea agradecer a Valeria Manzano y a los/las dos lectores/as anónimos/as por su atenta lectura y sus valiosas sugerencias. 
during the first years the journal focused on generational continuity as synonym of political virtue, later on it started to discuss about the merits of more iconoclastic attitudes by young people. The article stresses the heterogeneity of positions within this group of Italian communists, and their interest, and difficulty, to understand the new youth cultures.

Key words: Youth Cultures, 1960s, Italian Communist Party, History of Italy. 


\section{Introducción}

La aparición de una cultura juvenil en la Europa de los años ' 50 s y '60s constituye un tema frecuentemente visitado. Los estudios sobre la posguerra europea incluyen como una de las transformaciones más importantes de la época la emergencia de una fractura generacional que dividió al mundo de los recientes «teenagers» del de sus padres. Tanto la expansión del sistema educativo como las nuevas posibilidades de consumo por parte del segmento juvenil del mercado estimularon la visibilidad social y el sentimiento de unidad generacional por parte de adolescentes y jóvenes. Nuevos tipos de bailes y estilos musicales; cambios en las vestimentas y en la expresión corporal tales como el jean y el cabello largo; actitudes grupales y gregarias destinadas a reforzar la influencia de los pares generacionales por sobre la de los adultos; y posturas desafiantes o de malestar con respecto a la moral sexual predominante y roles de género tradicionales provocaron una grieta generacional difícil de pasar por alto para los contemporáneos. Para una generación que había madurado en épocas de crisis económica, guerra, y autoritarismo, el surgir de actitudes de desafiante jocosidad, irreverencia, y conspicuo hedonismo entre sus hijos apareció como un cambio difícil de procesar. ${ }^{2}$

La complejidad del mundo juvenil de posguerra se puede detectar a través de algunas paradojas. En primer lugar, en el hecho de que se conformaron verdaderas subculturas en el seno de la supuestamente homogeneizante sociedad de masas. De hecho, los y las jóvenes de los ' $50 \mathrm{~s} \mathrm{y} \mathrm{'} 60$ s diferenciaron sus gustos respecto al de generaciones mayores a través del consumo de formas de entretenimiento específicas como ciertos films, bailes, y ritmos musicales; o bienes que se convirtieron en indicadores de juvenilismo, como motonetas, zapatillas deportivas, o jeans. En segundo lugar, por la tensión entre una unidad identitaria juvenil, y la multiplicidad de subculturas que surgieron durante estas décadas, tales como teddy boys, mods, rockers, beats, hippies, y sus versiones nacionales, como los blouson noirs franceses, o los halbstarke alemanes. En tercer lugar, en la tensión entre identidades juveniles afirmadas en la esfera del consumo, el

2 Para historias generales del siglo xx que analizan el surgir de una cultura juvenil distintiva, ver por ejemplo Eric Hobsbawm, The Age of Extremes: A History of the World 1914-1991, Vintage Books, NY, 1994, pp. 323-329; Tony Judt, Postwar: A History of Europe since 1945, Penguin, NY, 2005, pp. 347-353 y 390-398; y Mark Mazower, Dark Continent: Europe's Twentieth Century Vintage Books, NY, 1998, pp. 316-319. 
entretenimiento, y el sistema educativo, y la creciente participación política por parte de grupos juveniles. De esta forma, a medida que se avanza en los años ' 60 s, las culturas juveniles pasan de ser consideradas como escapistas, superficiales, o indiferentes, a convertirse en verdaderas usinas de activismo político en torno a temas como el desarme nuclear o la oposición a la guerra de Vietnam, hasta devenir el centro de la cultura contestataria estudiantil de fines de los ' $60 \mathrm{~s}$. No por nada se ha referido a la situación de los jóvenes de posguerra como estando «entre Marx y CocaCola.» ${ }^{3}$

Lo disruptivo y contradictorio de las nuevas culturas juveniles dejó una profunda impresión en el mundo de los adultos. Es por eso que muchas de las aproximaciones a la juventud y sus transformaciones durante la posguerra enfoquen en las reacciones por parte de otros grupos sociales. De esta forma, en muchos casos se resalta el «pánico moral» y la criminalización de prácticas juveniles como una de las actitudes dominantes de la época. ${ }^{4}$ De la misma manera, los estudios sobre la juventud europea suelen resaltar los miedos sobre la creciente «americanización» cultural evidenciada en las nuevas generaciones. Dado que la mayoría de los consumos, formas de entretenimiento, y expectativas de vida de los jóvenes a partir de los '50s se conformaron en una cultura visual e informativa globalizada, muchos testigos de estos cambios los percibieron en términos de invasión cultural. ${ }^{5}$ Relacionados con estos temas está también la complicada relación entre la juventud y los partidos de izquierda tradicional. En este caso,

${ }^{3}$ Estudios específicos sobre casos nacionales enfatizando estos ejes, en Alex Schildt y Detlef Siegfred (eds), Between Marx and Coca-Cola: Youth Cultures in Changing European Societies, 1960-1980, Bergham Books, NY, 2006; Uta Poiger, Jazz, Rock and Rebels: Cold War Politics and American Culture in a Divided Germany, University of California Press, Berkeley, 2000; David Fowler, Youth Culture in Modern Britain, c 1920-1970, Palgrave McMillan, NY, 2008; Martin Klimke y Joachim Scharloth (eds), 1968 in Europe: A History of Protest and Activism, 1956-1977, Palgrave McMillan, NY, 2008.

${ }^{4}$ El texto clásico sobre el «pánico moral» como forma de recepción de los jóvenes es Stanley Cohen, Folk Devils and Moral Panics: The Creation of the Mods and the Rockers, Routledge, Londres, 2002 [1972] que analiza el caso de Gran Bretaña. Otro ejemplo, aunque para los EEUU de los '50s, es Luisa Passerini, «La Juventud, Metáfora del Cambio Social (dos Debates sobre los Jóvenes en la Italia Fascista y en los Estados Unidos durante los Cincuenta)» en Giovanni Levi y Jean-Claude Schmitt (eds), Historia de los Jóvenes: II La Edad Contemporánea, Taurus, Madrid, 1996, pp. 419-445.

5 Sobre el tratamiento de la «americanización» en el contexto especifico de las Alemanias de posguerra, ver Poiger, Jazz, Rock and Rebels. Para una visión más general, ver Judt, Postwar..., pp. 350-353. 
se suele resaltar el desencuentro entre organizaciones políticas centralizadas y demandantes de una disciplina verticalista, y una sensibilidad juvenil oscilante entre la indiferencia política o un activismo espontaneísta que no se llevaba bien con las maquinarias partidarias tradicionales. ${ }^{6}$

El presente trabajo busca contribuir a los estudios sobre culturas juveniles en Europa occidental a través del análisis de la emergencia de una «cuestión juvenil» en el seno de un semanario vinculado al Partido Comunista Italiano [PCI]: la revista Vie Nuove (1946-1971). Enfocando en los años 1960 a 1966 (es decir, dejando afuera del presente estudio el período abierto con los levantamientos estudiantiles de 1968), este artículo reconstruye diversas percepciones sobre la juventud por parte de un sector de la izquierda comunista italiana. Trabajar con esta fuente y en este periodo en particular presenta una serie de beneficios. En primer lugar, porque la revista Vie Nuove no era un estricto órgano partidario dirigido a militantes, sino que adoptaba el formato de una revista de actualidad dirigida a un público ideológica, social, y etariamente amplio. En este sentido, las percepciones y reacciones ante las actitudes, estilos y prácticas adoptados por los jóvenes de la época aparecen de manera vivencial y espontánea. Quienes expresan sus pareceres sobre los jóvenes en las páginas de Vie Nuove conforman un abanico de situaciones incluyendo desde amas de casa, docentes, comerciantes y, por supuesto, también jóvenes. Más aun, en la mayoría de los casos no opinan o hablan desde lugares de militantes o teóricos, sino a partir de vivencias cotidianas y percepciones espontáneas.

En segundo lugar, los años estudiados en este trabajo constituyen un momento de transición, en el que ciertas actitudes y características de la cultura juvenil aparecen a los comunistas italianos como plenas de ambivalencia. Por un lado, las formas de entretenimiento propiamente juveniles generan desconfianza y rechazo, puesto que se las ve como modos de escapismo, e incluso como ofensivas a ciertos principios de respetabilidad y decoro de los que los comunistas no eran refractarios. Por otro lado, se comienzan a ver rasgos de inconformismo e inquietudes que para muchos constituyen una invitación a la apertura y a la comprensión. Tal ambivalen-

${ }^{6}$ Para una visión general, ver por ejemplo Mazower, Dark Continent, pp. 318-319; y Geoff Eley, Forging Democracy: The History of the Left in Europe, 1850-2000, Oxford University Press, NY, 2002, pp. 341-357. Análisis más detallados sobre la ruptura entre movimientos estudiantiles o juveniles y las estructuras políticas tradicionales en Jan Kurz y Marica Tolomei, «Italy;» Martin Klimke, «West Germany;» Ingrid Gilcher-Holtey, «France,» en Klimke y Scharloth (eds), 1968 in Europe,pp. 97-110; 111-124; 125-136 respectivamente. 
cia no debería ser sorprendente, sobre todo si consideramos que los años sesenta italianos constituyen un período de transformaciones profundas y aceleradas, cuyo significado fue difícil de descifrar para los contemporáneos.

Entre fines de los '50s y mediados de los ' 60 s, de hecho, Italia comenzó el llamado «boom» o «milagro económico» mediante el cual el país pasó de ser una nación mayoritariamente agrícola y de bajos niveles de consumo, a constituirse en una sociedad urbana e industrial, con un significativo aumento del producto interno por habitante, migración de población rural hacia las ciudades del norte del país, y la aparición de nuevos empleos en industrias y servicios. Tales cambios vinieron acompañados de otros procesos cruciales. El acceso de las mujeres a nuevas profesiones, la educación superior, y su mayor participación en el mercado de trabajo es sin duda una de las más importantes. La expansión del sistema educativo, al elevarse la edad de la obligatoriedad escolar y el reforzarse la educación mixta fue también un cambio clave con consecuencias claras para la manera en que muchachos y muchachas conformaron nuevas experiencias e identidades en la Italia de los sesenta. Finalmente, el boom económico abrió también el camino a la conformación en Italia de una cultura de masas, a través del creciente rol de los medios de comunicación, la estandarización y unificación de gustos y estilos, y la proliferación de mensajes publicitarios orientados a un público general desprovisto de identidades clasistas o regionales. ${ }^{7}$

Todas estas transformaciones impactaron en una sociedad con una extensa lista de frustraciones políticas y sociales que agravaban distintos tipos de conflictos y antagonismos. De hecho, el «boom» afectaba solo a algunas capas de la población italiana. Pautas de bienestar tales como el acceso a los bienes de consumo durables, las vacaciones, y el trabajo estable eran beneficios muy selectivos, que convivían con amplios sectores que aún no habían podido resolver problemas de vivienda, o padecían realidades laborales de alta precarización. El descontento se extendía también

7 Para una descripción general de este proceso, ver Guido Crainz, Storia del miracolo italiano: Culture, identità, trasformazioni fra anni cinquanta e sessanta (Donzelli, Roma, 2005) 87-162; Paul Ginsborg, A History of Contemporary Italy: Society and Politics, 19431988, Palgrave, NY, 2003, pp. 210-297. Sobre los cambios en roles de género, ver Piccone Stella, Prima generazione: Ragazzi e ragazze del miracolo economico italiano, Franco Angeli, Milan, 1993, pp. 132-143. Sobre el surgir de la cultura de masas en Italia, ver John M. Foot, «Mass Culture, Popular Cultures and the Working Class in Milan, 1950-1970» Social History, vol. 24 (2), 1999; y Stephen Gundle, «L'americanizzazione del quotidiano. Televisione e consumismo nell' Italia degli anni cinquanta», Quaderni Storici, 62, 21, n 2, Agosto 1986. 
a otras áreas. Actitudes represivas, personal burocrático, e incluso legislación de la época del fascismo estaban todavía en vigencia; roles rígidos y patriarcales en la vida familiar y las relaciones de género tardaban en desaparecer; y el sistema político era percibido por muchos como corrupto y excesivamente lento para responder a las múltiples demandas (nuevas y postergadas) de la población. ${ }^{8}$

Al centrarse en Vie Nuove, este trabajo busca contribuir al estudio de la relación entre el comunismo italiano y la juventud. Dado que la mayoría de los estudios sobre el tema se concentran en la movilización estudiantil de fines de los ' 60 s, existen referencias solo generales a la manera problemática en que representantes del comunismo italiano percibieron el emerger de las culturas juveniles desde fines de los '50s. ${ }^{9}$ Estos estudios describen una situación ambigua. Reacciones hostiles hacia los nuevos estilos musicales y las pautas de consumo juveniles estuvieron ciertamente presentes en la prensa partidaria. En ocasiones esto expresaba temores de una creciente apatía juvenil hacia la política, o incluso una adhesión (a veces velada, a veces explícita) a patrones tradicionales de respetabilidad y decoro. Por otro lado, se menciona que, a pesar de tal hostilidad, en sus fiestas partidarias y gustos personales, los militantes comunistas no dejaban de rendir culto a los nuevos ídolos musicales del momento. Finalmente, también se menciona la manera en que los comunistas rechazaron los análisis más estigmatizadores acerca de la juventud contemporánea como una generación escapista e indiferente, buscando mostrar un perfil siempre optimista y esperanzador. ${ }^{10}$ Partiendo de estos análisis, este trabajo sugiere que habría que pensar las reacciones del comunismo italiano sobre la cultura juvenil como cambiantes y heterogéneas. Lejos de considerar que hubo una visión unificada a lo largo de los años, las siguientes páginas enfatizan que dentro de

${ }^{8}$ Algunas de las frustraciones más flagrantes consistían en lo lento del proceso de eliminación de la legislación heredada del fascismo, y su reemplazo por una más democrática. Ver sobre esto, Crainz, Storia, pp. 24-31.

9 Ver por ejemplo, Stephen Gundle, Between Hollywood and Moscow: The Italian Communists and the Challenge of Mass Culture, 1943-1991, Duke University Press, Durham, 2000, pp. 124-134; Diego Giachetti, Anni sessanta comincia la danza: Giovani, capelloni, studenti ed estremisti negli anni della contestazione, BFS, Pisa, 2002, pp. 163-232; Robert Lumley, States of Emergency: Cultures of Revolt in Italy from 1968 to 1978, Verso, Londres, 1990, pp. 47-142; Giuseppe Carlo Marino, Biografia del sessantotto: Utopie, Conquiste, Sbandamenti, Bompiani, Milan, 2003, pp. 324-342; Tolomei, «Italy.»

${ }^{10}$ Los análisis más completos en este sentido en Gundle, Between Hollywood and Moscow, 108-114; y Giachetti, Anni sessanta, pp. 9-14, 26-32, 83-85, 102, 151, 160-161. 
la comunidad de Vie Nuove existieron distintas percepciones e importantes desacuerdos sobre la cultura juvenil. Pensar la relación entre cultura juvenil y comunismo italiano es entonces acercarse a una realidad en donde las rápidas transformaciones de la primera se complementaban con la diversidad de miradas entre los simpatizantes del PCI.

Más allá de la heterogeneidad de posturas dentro de la revista, podemos diferenciar esquemáticamente dos momentos en cómo Vie Nuove se aproximó al tema de la juventud. En primer lugar, desde comienzos de la década de 1960 muchos observadores tendieron a interpretar todas las actitudes que diferenciaran a los jóvenes de los adultos como signos problemáticos de inmadurez o falta de un espíritu cívico. Como consecuencia de esto, los comunistas italianos enfatizaban la continuidad intergeneracional como el rasgo más sobresaliente de una juventud social y políticamente sana. Posteriormente, a partir de mediados de la década del 60, las percepciones se vuelven más complejas. El surgir de una contracultura juvenil que hacía hincapié en el inconformismo y se expresaba a través de movimientos o modas vinculadas a los beatniks norteamericanos, el pelo largo, o la música pop, abrieron un intenso debate en el seno de los comunistas de Vie Nuove. Si bien a muchos estas actitudes les parecían signos de rebeldía y genuino inconformismo, muchos otros las percibieron como una manera comercializada y alienada de canalizar energías políticas. Más aún, existieron también voces firmes e importantes que censuraron en términos conservadores y fuertemente moralistas las nuevas orientaciones juveniles. La conclusión del presente trabajo resalta la diversidad de percepciones dentro del comunismo italiano, así como subraya el hecho de que el PCI, o al menos esta publicación particular, buscó fomentar el debate amplio y sincero entre posiciones y puntos de vista profundamente divididos.

\section{Comunismo Italiano}

El PCI ocupa un lugar destacado dentro de la historia italiana de posguerra. Esto se debe, sin duda, a su relevancia como fuerza política. Si bien el PCI nunca llegó a formar un gobierno propio, se mantuvo a lo largo de la historia de la Italia republicana como la segunda fuerza electoral, conservando una constante de votos de entre el 20 y el $25 \%$ del padrón. A su vez, su estructura partidaria, su inserción sindical, y sus múltiples organizaciones de masas constituyeron al PCI en una corriente de importancia para la vida social italiana. Aparte de su impacto político, el 
PCI ha atraído la atención de historiadores e investigadores por su intenso trabajo cultural, vinculado a su búsqueda por insertarse capilarmente en el tejido social, así como a su esfuerzo en atraer intelectuales y operadores culturales de peso. ${ }^{11}$

Si bien sus orígenes se remontan a 1921 (cuando un grupo de activistas rompió con el Partido Socialista para formar el nuevo Partido Comunista Italiano), fue a partir de 1944 cuando el PCI comenzó la estrategia que lo transformó en un partido de masas. Durante ese año, su líder Palmiro Togliatti retornó del exilio en la Unión Soviética y delineó la política que caracterizaría al partido durante los años posteriores a la caída del fascismo. ${ }^{12}$ La fórmula fundamental consistió en renunciar a una vía insurreccional hacia el socialismo, en favor de una política de alianzas y consensos con todas las fuerzas políticas democráticas. Los objetivos de la revolución socialista fueron relegados en favor de la búsqueda de la liberación nacional (en un momento en que parte de Italia todavía estaba ocupada por la Alemania Nazi), la coordinación de la política anti-fascista (permitiendo así el fortalecimiento y la unidad de los grupos de la Resistencia), y la gradual conquista de una «democracia progresiva» que garantice mayor participación cívica y bienestar social. Esta estrategia se extendió en los años de posguerra, cuando el PCI colaboró e impulsó la asamblea constituyente que creó las principales instituciones de la república italiana, lo cual lo llevó a consolidarse como un partido de masas abocado a la profundización de la democracia italiana, y reputado por su compromiso con la Resistencia.

11 Por esta razón, la bibliografía son la historia del PCI es vasta y heterogénea. Para la historia del PCI dentro de narrativas generales de la historia italiana contemporánea, ver Ginsborg, A History of Contemporary Italy, pp. 44-48,79-88, 195-209; 290-297; Silvio Lanaro, Storia dell'Italia republicana: La economia, la politica, la cultura, la società dal dopoguerra agli anni '90, Marsilio, Venezia, 1992, pp. 68-87; y Crainz, Storia del miracolo italiano, 33-56, 242-245. Para estudios específicos sobre el PCI: Alexander De Grand, The Italian Left in the Twentieth Century: A History of the Socialist and Communist Parties,Indiana University Press, Bloomington, IN, 1989, pp. 81-163. Una perspectiva del PCI en el marco del comunismo y el socialismo en Europa en Donald Sassoon, One Hundred Years of Socialism: The West European Left in the Twentieth Century, Harper Collinis, Londres, 1997, pp. 73-82, 102-106, 129-130, 260-263, 301-304. Los estudios más recientes apuntan a aspectos específicos sobre la política cultural o temas atinentes a la construcción de género, intimidad, y privacidad. Ver Gundle, Between Hollywood and Moscow; y Sandro Bellasai, La Morale Comunista. Pubblico e Privato nella Rappresentazione del PCI, 1947-1956, Carocci, Roma, 2000.

12 Ginsborg, A History of Contemporary Italy, pp. 44-48; 79-88; Sassoon, One Hundred Years of Socialism, pp. 73-82, 102-106. 
La política de unidad nacional, y su tendencia a las alianzas y la moderación le permitió al PCI adaptarse al sistema político de la Italia republicana, y romper el aislamiento durante los años de la guerra fría. Esto no fue poco, especialmente considerando las importantes presiones desde la Iglesia, el sector empresarial, y los EEUU en favor de marginar, e incluso ilegalizar al PCI. Ganarse un lugar dentro de la política oficial, sin embargo, implicó postergar importantes demandas en torno a la reforma agraria, el reclamo de mayor poder y libertad sindical, y el compromiso con reformas atinentes a la profundización de la democracia. Como fondo de esta situación se encontraba una encrucijada crucial de la política del PCI: mantenerse fiel e intransigente con respecto a demandas provenientes de la militancia fabril y el activismo obrero y campesino, o buscar posturas que le permitieran ampliar su base de votantes. La resultante de esta tensión fue el buscar un lugar dentro del sistema de partidos a través del mantenimiento de una estructura partidaria verticalista, el fomento del mito de la Unión Soviética como forma de cohesionar a sus adherentes, y el despliegue de una sensibilidad especial para ganar aceptación y respetabilidad dentro del clima cultural de la guerra fría. ${ }^{13}$

Junto con su planteo político, el PCI se caracterizó también por su vigor organizativo, evidenciado en su estrategia de construcción «capilar» mediante la cual buscó expandirse socialmente. Los locales partidarios se encontraban hasta en los pueblos más pequeños y remotos, compitiendo así con la presencia local de la Iglesia y el principal rival político: la Democracia Cristiana [DC]. En los barrios clásicos de la clase obrera industrial los comités del PCI se convertían en espacios cruciales de la sociabilidad de sus habitantes. Otros espacios característicos, como las «casas del pueblo» que pululaban a lo largo del país sostenidas por iniciativas locales, se volvían lugares de recreación y activismo cultural. Lo mismo puede decirse de festividades como las «fiestas de La Unidad,» [feste dell'Unitá] en las cuales se congregaba a militantes y simpatizantes en torno a jornadas recreativas orientadas a juntar fondos para el diario partidario L'Unitá. Otras asociaciones partidarias, como la Udi [unione donne italiane/unión de mujeres italianas], buscaban integrar el activismo femenino a través de una política amplia, destinada a incorporar a mujeres de izquierda más allá de las líneas estrictamente partidarias y buscando inte-

${ }^{13}$ Ginsborg, A History of Contemporary Italy, pp. 79-88; 118-120; 195-204; Lanaro, Storia dell'Italia repubblicana, pp. 68-87. 
grar demandas de género a la política nacional a través sobre todo de su periódico Noi donne. ${ }^{14}$

La vasta estructura organizativa del PCI fue también crucial para desplegar su política de acercamiento y cooptación de los intelectuales. Como parte de la estrategia de ganar respetabilidad y extender su alcance más allá de los exclusivos límites de la clase obrera industrial, el PCI, y especialmente Togliatti, desplegó una política de cortejo y adulación de los intelectuales, entendiendo que los mismos desarrollaban una función crucial para la integración social y la creación de un bloque unificado de fuerzas progresistas. El PCI fue de hecho el partido italiano más abocado a incorporar a sus filas a cineastas, escritores, artistas plásticos, ensayistas, filósofos, profesores universitarios, historiadores, y otros hombres de letras. A través de revistas, casas editoriales, e institutos culturales, el PCI pudo aparecer para muchos intelectuales como la única posibilidad en la Italia de posguerra de crear un espacio cultural laico, progresista, y popular. Los atractivos del PCI, sin embargo, no hicieron desaparecer los problemas, especialmente en relación con la tensión entre la disciplina partidaria y la búsqueda de mayor autonomía para el campo cultural. De allí que durante muchos años el PCI funcionara como un espacio de atracción de intelectuales anti-clericales de izquierda, que sin embargo evidenciaron su malestar con lo que consideraban la estrechez de miras y el dogmatismo de los principales dirigentes culturales del comunismo italiano. ${ }^{15}$

14 Gundle, Between Hollywood and Moscow, passim; Bellasai, La Morale Comunista. Sobre comunismo, cuestiones de género, y el movimiento de mujeres, ver Giulietta Ascoli, «L'UDI tra emancipazione e liberazione», Problemi del socialismo, 17 (4), 1976, 109159; Lesley Caldwell, Italian Family Matters: Women, Politics and Legal Reform. Macmillan, Londres, 1991; María Casalini, «The family, sexual morality and gender identity in the communist tradition in Italy (1921-1956)», Modern Italy, 18 (3), 2013; Yasmine Ergas, «Feminism and the Italian party system: Women's Politics in a Decade of Turmoil,» Comparative Politics, 14, 3, 1982, 253-279; Stephen Gundle, «Femminine Beauty, National Identity and Political Conflict in Postwar Italy, 1945-195», Contemporary European History, 8 (3), 1999; Nina Rothemberg, «The Catholic and the Communist Women's Press in Post-War Italy - An Analysis of Cronache and Noi Donne», Modern Italy, 11 (3), 2006; Mark Seymour, «Steel Capsules and Discursive Monopolies. 'Noi Donne' and Divorce in Italy», Storicamente, 6, 2010.

15 Para un análisis de estas politicas vér Gundle, Between Hollywood and Moscow, 11-41; Albertina Vittoria, «La commissione culturale del PCI dal 1948 al 1956,» Studi Storici, 31 (1), 135-170; y David Forgacs, L'industrializzazione della cultura italiana, Il Mulino, Bolonia, 2000, pp. 233-264. 
La vasta red de comités, clubes, asociaciones juveniles, y periódicos del PCI fueron cruciales para generar una subcultura que permitió reforzar el compromiso y la solidaridad entre sus miembros, y sostener la influencia partidaria en la vida cotidiana de los militantes. Una característica crucial del PCI fue de hecho su intensa labor pedagógica, centrada fundamentalmente en la educación política de sus militantes y adherentes. Esta esfera de la actividad ha sido recientemente estudiada con interés, puesto que implicaba el asumir posicionamientos y debates sobre un vasto campo de temas, incluyendo asuntos de la esfera privada, las relaciones de género, la vida cotidiana, la familia y la «moral.» ${ }^{16}$ De esta forma, el PCI se constituyó en una importante fuerza orientada a generar una nueva cultura comunista que se extendía hasta todas las esferas de vida de sus miembros. Este es un aspecto que revela el carácter voluntarista de los militantes y dirigentes del PCI, sobre todo si se tiene en cuenta lo ambicioso de este planteo: el buscar consolidar una cultura alternativa en tiempos en que la Iglesia católica mantenía su poder y las instituciones de la cultura de masas comenzaban a evidenciar su influencia.

Mientras que ciertas características generales se mantuvieron estables durante gran parte de la historia del PCI, existieron contextos particulares que implicaron crisis importantes. El año 1956 fue el más claro de ellos, cuando los comunistas italianos debieron afrontar el desafío constituido por las revelaciones del XX Congreso del Partido Comunista Soviético acerca de las prácticas dictatoriales del estalinismo. ${ }^{17} \mathrm{Si}$ consideramos que en su artículo necrológico de 1953, el periódico L'Unità había definido a Stalin como «el hombre que más ha hecho por la liberación del género humano,» no es difícil imaginar el impacto de tales revelaciones sobre uno de los emblemas del comunismo italiano. ${ }^{18}$ Para complicar más las cosas, ese mismo año la Unión Soviética invadió Hungría, lo cual hundió aún más las fuentes de prestigio del PCI (que avaló la intervención). El impacto, de hecho, fue tremendo, e implicó la pérdida de tal vez hasta 400.000 militantes, rupturas de notorios intelectuales, y expulsiones o renuncias de importantes dirigentes. Sin embargo, más allá de estas pérdi-

16 Ver sobre todo Bellasai, La Morale Comunista.

17 Todos los estudios sobre el PCI refieres a esta crisis. Ginsborg, a History of Contemporary Italy, pp. 204-209; Sassoon, One Hundred Years of Socialism, pp. 260-263; Marc Lazar, «Les partis communistes italien et francais et l'après-Stalin,»Vigtième Siecle, 28, 1990; y Vittoria, «La commissione culturale del Pci dal 1948 al 1956,» pp. 157-170.

${ }^{18}$ La cita de L'Unità en Lanaro, Storia dell'Italia republicana, p. 68. 
das, el PCI logró superar el trauma manteniendo la unidad. En parte esto se debió a una política de creciente distanciamiento con respecto a Moscú, mediante la cual Togliatti buscó deshacerse del servilismo y adulación anteriores. Al mismo tiempo, la crisis de 1956 aceleró la renovación generacional, dando lugar a una nueva camada de dirigentes más interesados en mostrar su adhesión a las libertades democráticas como un valor en sí mismo (y no tanto por su utilidad táctica). Fue así como el PCI atravesó el año 1956 manteniendo su caudal electoral y su status como el principal partido de masas de la izquierda italiana.

Luego de la crisis de 1956, el PCI atravesó otro momento de incertidumbre y replanteos a mediados de los ' $60 \mathrm{~s} .{ }^{19}$ Diversos factores se coaligaron para ello. En primer lugar, la coyuntura política aportaba un panorama complicado. Desde fines de los 50s, la DC había iniciado un «giro a la izquierda» en alianza con el Partido Socialista, lo cual se concretó en una serie de reformas vinculadas a la nacionalización de la energía, la expansión de la educación media, y la introducción de regulaciones al sistema financiero. Paradójicamente, el abandono del conservadurismo por parte de la DC significó un importante desafío para el PCI, ya que quedó políticamente aislado cuando los socialistas se sumaron a la coalición gobernante. Más aun, el PCI se veía ahora en la encrucijada de tener que mostrarse como un férreo opositor al gobierno en nombre de una alternativa verdaderamente radical, mientras que debía evitar obstaculizar las reformas emprendidas por la nueva coalición. En segundo lugar, la súbita muerte de Togliatti en 1964 reabrió divisiones y pujas por la sucesión que complicaron la unidad partidaria. Finamente, las transformaciones más profundas de los 60s implicaron un desafío al PCI. Si bien el caudal electoral del comunismo italiano se mantenía estable (y a veces incluso superaba el 25\%, como en el caso de las elecciones de 1963), y su presencia mediática se afianzaba (L'Unità era el diario más leído en los $60 \mathrm{~s}$, luego del Corriere della Sera), la militancia partidaria decrecía (de 2.035.000 en 1956 a 1.576 .000 en 1966). ${ }^{20}$ Más preocupante aún, la edad promedio de los militantes ascendía, lo cual prendía la luz de alarma acerca de la capacidad del PCI para ganar a las nuevas generaciones y adaptarse a las nuevas realidades de la moderna sociedad de masas. En este sentido, debates y polémicas en torno a la juventud, las características de la sociedad de

19 Ginsborg, A History of Contemporary Italy, 290-295; Sassoon, One Hundred Years of Socialism, pp. 301-304.

20 Ginsborg, A History of Contemporary Italy, pp. 290-291. 
consumo, y las modalidades del cambio social en la Italia de posguerra se volvían cruciales para mantener la vigencia de un partido que, a lo largo de los años, se había ganado el puesto de ser la «organización comunista más vasta del mundo occidental.» ${ }^{21}$

\section{El fantasma de la despolitización}

Durante los primeros años ' 60 , la aproximación de Vie Nuove al tema de la juventud estuvo signada por un fantasma que recorría los distintos artículos: la despolitización, la pérdida del compromiso cívico, o lo que se percibía como una creciente desideologización entre las nuevas generaciones. Desde fines de la década de 1950, de hecho, sociólogos, periodistas, psicólogos, pedagogos y observadores varios coincidieron en construir tres imágenes centrales que resumían las características de la juventud del momento. ${ }^{22}$ La primera describía a la juventud como un grupo social caracterizado por el creciente conformismo, la búsqueda de ascenso social, el pragmatismo, la postergación de los valores cívicos y el compromiso ideológico y, sobre todo, el acceso al consumo como el principal indicador de status social y satisfacción personal. De acuerdo con esta imagen, la juventud italiana de los años '50s y tempranos ' 60 s se podía resumir como la generación de las tres «m»: macchina, moglie e mestiere (auto, esposa y oficio). En segundo lugar, no fueron pocos los que notaron que el auge del consumismo se combinaba con la creciente «americanización» de gustos y costumbres por parte de las nuevas generaciones. Con la expansión de los medios de comunicación de masas, la influencia de las películas de Hollywood, y el creciente rol de la publicidad, la asimilación de americanización y juventud fue difícil de soslayar. De esta manera, el

${ }^{21}$ Idem, p. 291.

${ }^{22}$ Reproduzco aquí los principales estudios sobre el tema a cargo de Simonetta Piccone Stella, Prima generazione, pp. 9-112 y 145-180; idem, «All'origine della cultura giovanile negli anni sessanta: transgressività, esibizionismo, nuovi consumi», Problemi del Socialismo, 13, 1991; idem, «'Rebels without a Cause': Male Youth in Italy around 1960», History Workshop, 38, 1994; Diego Giachetti, Anni sessanta comincia la danza; Crainz, Storia del miracolo italiano, pp. 75-84; Alessandro Portelli, «L'orsachiotto e la tigre di carta. Il rock and roll arriva in Italia», Quaderni Storici, 58, 1985; e idem, «Luigi's Socks and Rita's Makeup: Youth Culture, the Politics of Private Life, and the Culture of the Working Classes,» en Alessandro Portelli, The Battle of Valle Giulia. Oral History and the Art of Dialogue, University of Wisconsin Press, Madison, 1997, pp. 232-248. 
impacto de nuevos estilos musicales como el twist y el rock and roll, y la difusión de productos como la Coca Cola o los blue jeans constituyeron marcas más que visibles del intenso vínculo entre cultura juvenil, influencia americana, y modernización socio-cultural. En el marco de la guerra fría y de la decidida oposición del PCI al alineamiento con los Estados Unidos y el Plan Marshall, esta última característica aparecía como altamente problemática a los distintos observadores del comunismo italiano.

Si el conformismo y la americanización eran alarmantes para los comunistas, la tercera imagen sobre la juventud circulante en esta época era igualmente problemática: se trataba nada menos que de un difundido "pánico moral» acerca de la creciente criminalidad juvenil. Desde fines de la década del ' 50 se acrecentó el temor y el alarmismo con respecto a delitos como robo de autos, peleas callejeras, o atentados contra la propiedad por parte de grupos de jóvenes. Dado que este tipo de crímenes no estaba vinculado a un móvil económico inmediato y que en muchos casos involucraba a jóvenes de familias estables y lejanas del mundo de la marginalidad, pronto se difundió la idea de que este tipo de actos era producto de la falta de valores de una generación que atravesaba un proceso de modernización acelerada en donde la estructura familiar se ponía en entredicho. No era casual, de hecho, que entre las razones que se aducían para explicar la delincuencia juvenil figuraran la migración y el trauma cultural que ésta acarreaba a jóvenes procedentes de las regiones rurales del sur de Italia al encontrarse solos en las ciudades del norte.

Conformismo, americanización y criminalidad eran ciertamente perspectivas poco esperanzadoras para los comunistas italianos, quienes veían a la vez cómo se despoblaban de jóvenes sus filas de militantes. Mientras en 1956 los organismos juveniles del PCI contaban con alrededor de 358.000 miembros, para 1966 su número era de $154.000 .^{23}$ Frente a esta situación, las principales reflexiones sobre la juventud en Vie Nuove buscaban hacer un doble juego: atribuir los rasgos negativos de los jóvenes a los males de la sociedad capitalista; y enfatizar que, a pesar de tales tendencias, la mayoría de los jóvenes italianos permanecía comprometida a ideales políticos participativos y democráticos. De esta forma, gran parte del desinterés y la indiferencia de los jóvenes frente a la política y al mundo de los adultos eran explicadas por la incapacidad de la sociedad

${ }^{23}$ Ver Ginsborg, A History of Contemporary Italy, p. 290 y Gundle, Between Hollywood and Moscow, p. 113. 
capitalista para incorporar a la juventud a un proyecto motivador. La idea de que la juventud italiana estaba expuesta al «miedo de un mañana incierto, sin perspectivas» era invocada como explicación general de la apatía juvenil. ${ }^{24}$ Estos análisis eran reforzados por testimonios de los mismos jóvenes, enfatizando la vinculación entre frustración social y violencia. Tal cual explicaba un joven lector respondiendo a una encuesta sobre el tema de la juventud,

Para mí, un joven que después de ocho horas de trabajo por cuatro pesos se encierra en un cine de barrio (...) o que pasa las horas jugando a las cartas es un imbécil. Antes que él prefiero los teddy boys que junto a los amigos se divierten destruyendo los carros de los burgueses o molestando a las tristes parejas de los enamoraduchos de periferia. ${ }^{25}$

Por un lado, entonces, no era difícil para los comunistas explicar la apatía y la violencia juvenil como consecuencia de los males sociales capitalistas e incluso como una forma de resistencia frente a los mismos. Sin embargo, esta estrategia implicaba aceptar el supuesto de la despolitización por parte de los jóvenes. Mucho más importante para los comunistas italianos era en cambio presentar un contra-modelo en el cual la juventud contemporánea era retratada como ejemplo de compromiso con propuestas políticas democráticas y transformadoras. En cierta manera las circunstancias políticas favorecían esta actitud. En junio de 1960, de hecho, el escenario político italiano fue sacudido por un levantamiento popular en contra del llamado «gobierno Tambroni,» el cual se había constituido pocos meses antes mediante una alianza parlamentaria entre la gobernante DC y el partido neo-fascista Movimiento Social italiano [MSI]. Los episodios de agitación callejera en Génova y otras ciudades en contra del gobierno duraron varios días, a pesar de la brutal represión gubernamental que provocó la muerte de varios manifestantes. Cuando a principios de Julio la principal organización obrera (la CGIL) llamó a una huelga general de alcance nacional y altísimo acatamiento, la situación del gobierno se debilitó a tal punto que debió renunciar y proceder a la formación de

${ }^{24}$ Miriam Mafai, «Primo: la paura del domani», Vie Nuove, n. ${ }^{\circ}$ 4, 23 de Enero de 1960. Para un análisis de los artículos de Vie Nuove_durante 1960 véase Giachetti, Comincia la danza, pp. 9-12. 1960 .

${ }_{25}$ Mario B, «La politica a che la fa di mestiere,» Vie Nuove, n. ${ }^{\circ}$ 8, 20 de Febrero de 
una nueva alianza sin los neo-fascistas. Las jornadas de junio y julio son generalmente referidas como un signo crucial de la fuerza del «consenso antifascista» dentro de la Italia republicana, ya que dejaron asentado que la población no iba a aceptar una reivindicación del pasado fascista por parte de la clase política. ${ }^{26}$ Además de ser un hecho político relevante, los observadores de la época no dejaron de advertir algo notorio: los principales protagonistas de los levantamientos callejeros eran jóvenes. ${ }^{27}$

El entusiasmo de los redactores de Vie Nuove con el súbito aparecer de jóvenes en la política (y en las calles) fue notorio. Los números correspondientes a esas semanas incluyen tapas, títulos, crónicas, coberturas fotográficas y mesas redondas sobre las revueltas callejeras que resaltan la participación y el protagonismo juvenil en la «lucha anti-fascista.» ${ }^{28}$ Una de las notas centrales sobre la revuelta en Génova, por ejemplo, abría con descripciones de adolescentes tirando piedras contra la policía y sumándose a las columnas de las organizaciones de ex-partisanos, a quienes protegían armando un cordón humano entre ellos al tiempo que entonaban viejos cánticos de la Resistencia. Tras la elocuente imagen, el cronista no podía dejar de concluir con una reflexión emocionada y esperanzadora acerca de las nuevas generaciones, y desacreditar al mismo tiempo las representaciones más negativas o escépticas sobre la juventud contemporánea:

La intervención de los jóvenes, que ha hecho posible el éxito, el impulso incluso violento, pero nunca desconsiderado, el sentimiento profundamente unitario de estos muchachos [ragazzi] revelan que algo muy importante ha cambiado. Algo que pertenece a la generación que, incluso sin haber conocido el fascismo, se lanza y lo combate al lado de

${ }^{26}$ La chispa que desató la explosión comenzó cuando el MSI decidió realizar un congreso nacional en la ciudad de Génova, lo cual generó la indignación de la población. Para detalles sobre este acontecimiento ver Ginsborg, A History of Contemporary Italy, pp. 256-258; Crainz, Storia del miracolo, pp. 169-179.

27 Sobre la participación juvenil en las jornadas de Junio y Julio de 1960, véase Crainz, Storia, pp. 180-188; y Giachetti, Anni sessanta, pp. 16-20.

28 Ver por ejemplo, Silvio Micheli, «Nemici vecchi unità nuova,» y G. F. P, «Anche a Palermo i giovani in prima fila,» Vie Nuove, n 28, 9 de Julio 1960; y la mesa redonda «I giovani: Perché vogliono un altra Italia,»Vie Nuove, n 30, 23 de Julio de 1960. El entusiasmo por la participación política juvenil se registra también en dos notas del escritor Pier Paolo Pasolini, quien tenía una columna fija titulada «Diálogos con Pasolini» en la que contestaba cartas de lectores sobre temas varios. Véase por ejemplo Pier Paolo Pasolini, «Il risveglio dei giovani,»Vie Nuove, n 29, 16 de Julio de 1960; e idem, «Ancora sui giovani,» idem, n 31, 30 de Julio de 1960. 
la generación de la Resistencia, para defender aquellos mismos valores de fondo que caracterizan la estación más bella de la historia de Italia. Pensaba en cómo vienen frecuentemente configurados los jóvenes. La amargura, la desconfianza, la ironía con la cual muchas veces se habla de los jóvenes quedan de lado frente a la explosión del joven estudiante y su participación junto a sus coetáneos obreros en la lucha contra el regurgito fascista. ${ }^{29}$

Las escenas y las instancias que les permitían confirmar la continuidad entre los jóvenes contemporáneos y la generación de la resistencia anti-fascista de la entre-guerra fueron para los editores de Vie Nuove una suerte de clímax que espantaba miedos sobre la despolitización y apatía juveniles. En los siguientes años, de hecho, las referencias a la juventud en la revista enfatizaron su aspecto cívico y politizado, reduciendo o mitigando los signos de desencuentro generacional. Esto se puede apreciar sobre todo en una larga investigación especialmente dedicada a la juventud publicada en junio de 1964 bajo el título de «nacidos después del diluvio,» algo que hacía referencia al hecho de ser la primera generación que no había conocido la guerra y el fascismo. La encuesta, realizada entre 1412 jóvenes de edades no precisadas y cuya proximidad al comunismo en muchos casos era difícil de disimular, arrojaba una conclusión más que optimista sobre las nuevas generaciones: a través de distintos testimonios y análisis de supuestos expertos en la cuestión, la encuesta describía a los jóvenes como personajes cívicamente comprometidos e interesados en dedicar su tiempo libre a actividades sanas como la lectura o la militancia política. ${ }^{30}$

En este marco, los conflictos y el malestar expresados por los jóvenes eran entendidos como producto de una profunda sensibilidad e inquietud social, y no como expresiones de apatía, indiferencia, o sumisión a las modas americanas. La encuesta, por ejemplo, reproducía entrevistas y testimonios de estudiantes secundarios quejándose por la frialdad y falta de motivaciones del sistema educativo, resaltando aquellos fragmentos en que los estudiantes reclamaban más interés de la escuela por la historia

${ }^{29}$ Silvio Micheli, «Nemici vecchi unità nuova.». De la misma manera procedía el editorial de Vie Nuove luego de una marcha multitudinaria en Palermo, protagonizada en gran parte por jóvenes. Para el editorialista, esto reflejaba «una continuidad de consciencia que deshace muchos mitos interesados sobre la «sordera» de las nuevas generaciones.» L Pa, «Il paese rifiuta la 'tregua,'»Vie Nuove, n. ${ }^{\circ} 28,9$ de Julio de 1960, p. 3 .

30 Guido Vicario, «La parola ai ventenni»Vie Nuove, n. ${ }^{\circ}$ 24, 11 de Junio de 1964. 
italiana reciente, o más libertades para la organización política estudiantil. ${ }^{31}$ Algo análogo sucedía con la porción de la encuesta dedicada a los jóvenes trabajadores, que aparecían como críticos de los sindicatos y sus internas, y se quejaban de que los viejos obreros no querían discutir de política con ellos, o los menoscababan cuando daban sus opiniones. Sin embargo, más allá de estas quejas, la mayoría de los entrevistados parecía coincidir en su malestar con respecto a las condiciones de trabajo y la necesidad de unidad y acción entre los obreros. ${ }^{32}$ Esto parecería coincidir con la opinión de un viejo sindicalista que ofrecía su experimentada perspectiva sobre la juventud actual. De acuerdo al dirigente obrero, los jóvenes eran ciertamente desorganizados, poco afectos a respetar la metodología del sindicato, e incluso políticamente ingenuos. Sin embargo, a la hora de la acción y la huelga estaban más que prontos a sumarse y participar «en la primera línea.» ${ }^{33}$

A pesar de la evidente intención por delinear un retrato en positivo de la nueva generación, no dejaban de aparecer nubarrones y sombras que retrotraían a los temores acerca de la apatía e indiferencia juvenil. En la introducción a la encuesta, por ejemplo, el presentador hacía referencia a la nueva cultura juvenil, a la música y a los nuevos ídolos de masa que absorbían el interés y las pasiones juveniles. Pero consideraba el fenómeno algo transitorio, producto de una sociedad en rápida trasformación que obligaba a los jóvenes a detener su madurez por un rato, hasta finalmente decidirse a entrar en el mundo de los adultos. ${ }^{34}$ Dentro de la encuesta, sin embargo, algunos participantes adoptaban un tono más pesimista. Tal era el caso del antropólogo Ernesto De Martino, quien observaba que la caída de los valores tradicionales y la ruptura de los jóvenes con el mundo de sus padres y sus familias podía, en algunos casos, llevar a «un individualismo exacerbado en las propias decisiones y en las relaciones con los otros jóvenes y con los adultos.» Esta especie de zambullida hacia un vacío de valores o de marcos contenedores era también lo que explicaba, según De Martino, la atracción de los jóvenes por el consumo y los productos de la sociedad de masas, y en especial lo que él definía como «la idolatría del motor, el agruparse en bandas, el circular en ruidosas moto-

31 Véase la mesa redonda «A scuola con i fantasma,» idem; y «Quando sono in clase mi pare di stare sola», idem.

32 «Dibattito: la fabbrica: Cominciare dai giovani,» idem.

${ }^{33}$ Luciano Lama (sindicalista), «In prima linea» idem.

34 Guido Vicario, «La Parola ai ventenni,» cit. 
cicletas, el vértigo de la velocidad.» Sobre todo, De Martino apuntaba a la seducción de los productos de la sociedad de masas como una forma de substitución de necesidades más sustanciales,

En la medida en que las viejas creencias caen, la necesidad de creer en cualquier cosa busca satisfacción en mitos estrechos, ficticios, como son esos que la sociedad ofrece a través del divismo cinematográfico y deportivo. (...) El joven deviene inconscientemente prisionero de estos mitos, en la medida en que puede satisfacer una exigencia no eludible y que la sociedad contemporánea no satisface: la de darse una esperanza, un fin en la vida. El fin podrá ser el jugador de fútbol, el púgil famoso, la diva del momento, la cantante de éxito: una mitología exclusiva y fanática, completamente privada de cualquier contenido ideal que denuncia un vacío en nuestra sociedad. ${ }^{35}$

Semejante conclusión chocaba fuertemente con el tono principal de la investigación, que era de un optimismo esperanzador. La encuesta, de hecho, incluía una votación sobre las figuras que los participantes consideraran más influyentes y modélicas. Albert Einstein figuraba primero con 337 votos, seguido muy de cerca por Fidel Castro, con 305. Bastante más abajo se situaba el papa Juan XXIII con 252 votos. Recién el cuarto puesto era para un cantante, Bobby Solo, con 53 votos, aventajando así al recientemente asesinado John F. Kennedy, con 41 votos, y al futbolista Gianni Rivera, con 35. ${ }^{36}$ Los resultados de la encuesta, por lo tanto, no parecían corroborar el alarmismo de De Martino acerca de la fascinación juvenil por el divismo hollywoodense y deportivo. Sin embargo, las palabras del antropólogo eran tal vez indicativas de que incluso cuando buscaban contrarrestarlas, la prensa comunista no dejaba de reproducir visiones fuertemente peyorativas, pesimistas, y alarmistas con respecto a la juventud contemporánea y su absorción por la cultura de masas.

De hecho, poco después de la encuesta tuvo lugar un intenso debate en la sección de cartas de lectores de la revista. ${ }^{37}$ El debate comenzó cuando

35 Ernesto De Martino, «Acqua guasta nel deserto» Vie Nuove, Ibid. Sobre la relación entre De Martino y el PCI ver Gundle, Between Hollywood and Moscow, pp. 59-61.

36 «Chi preferiscono,» Vie Nuove, n. ${ }^{\circ} 24$, cit.

37 Ver Vie Nuove, 36, 38, 40, 43, correspondientes al 3 y 17 de Septiembre y 1 y 22 de Octubre de 1964 respectivamente. Se trataba de cartas de lectores divididas en dos grupos: uno referido a los jóvenes, y el otro a la nueva música juvenil y en especial a las letras de las canciones consideradas vacías y sin argumento. Si bien los editores aseguraban que 
varios lectores escribieron a la redacción señalando que, si bien apreciaban los perfiles de los jóvenes retratados en la encuesta, sus experiencias cotidianas mostraban algo totalmente distinto. Un profesor de gimnasia de un colegio secundario de Roma, por ejemplo, se quejaba del «espantoso vacío del discurso» de sus alumnos, y señalaba que «jamás que recuerde les escuché mencionar, en su continuo charlar, el nombre de un escritor moderno, de un disco de música sinfónica, de una ópera, o la mínima referencia a acontecimientos internacionales como la crisis en Chipre o en Tonkín.» ${ }^{38}$ Lejos de ser un lamento aislado, quejas similares fueron pronto reproducidas por otros participantes. Un instructor de Liceo jubilado invocaba la imagen de la inmediata posguerra cuando, según él, sus estudiantes rebozaban de interés político y de buen gusto artístico, reflejado en sus preferencias por los autores del neorrealismo cinematográfico italiano tales como Visconti, De Sica y Rossellini. A esta imagen contraponía un poco feliz retrato de la juventud contemporánea: «hoy, si la TV transmite 'Tribuna política,' un concierto, o un informe especial, se puede estar seguro que los jóvenes huyen. Si se les pide noticias de un premio literario, no hay forma de sacarles una palabra de la boca (...) No saben una jota de política. En suma, un cuadro verdaderamente desolador.» ${ }^{39}$

Estas visiones no eran monolíticas, y fueron intensamente debatidas por otros lectores, especialmente los más jóvenes. Sin embargo, las posiciones encontradas no llegaban a cuestionar los términos del debate. En otras palabras, quienes defendían a la juventud contemporánea lo hacían bajo la fórmula de «no todos los jóvenes son así,» o victimizándolos bajo el signo de «es la sociedad la que los convierte en eso,» siendo entonces que a ninguno de los participantes se le ocurría señalar que tal vez los criterios mismos de juicio eran demasiado cerrados o tradicionales. ${ }^{40}$ Más aún, no faltaban los casos de lectores jóvenes que con una arrogancia bastante inocultable coincidían en condenar a su generación, reservándose

\footnotetext{
las cartas llegadas a las revistas eran una multitud, solo publicaron partes de 12 cartas a lo largo de estas semanas. La preocupación del semanario por la juventud se puede también observar en una entrevista a Jean Paul Sartre de fines de 1964, especialmente dedicada al tema. Ver «I giovani, sono cinici? Intervista con Jean Paul Sartre»Vie Nuove, n. ${ }^{\circ}$ 51, 17 de Diciembre de 1964, pp. 24-34.

38 «Ma insomma, come sono questi giovani?»Vie Nuove, n. . 36, 3 de Septiembre de 1964

39 idem.

40 Véase por ejemplo «lettere,» n. ${ }^{\circ} 40$ y n. $.^{\circ} 43,1$ y 15 de Octubre de 1964.
} 
para ellos mismos el rol de ser una elite político-cultural en un mar de mediocridad. Así entonces un joven militante observaba que

en la sociedad actual es muy difícil para un joven mantenerse en una conducta moral y cultural de cierto nivel. Todo es impostado de modo tal que solo los más fuertes acceden a quedar afuera del círculo vicioso y martillante de los discos, de la publicidad, de la necesidad de cosas inútiles. ${ }^{41}$

A mediados de 1964, por lo tanto, las páginas de Vie Nuove reflejan una importante tensión dentro de un consenso general. Por un lado, mientras los editorialistas trataban de convencer a los lectores sobre las virtudes políticas y morales de la nueva generación, muchos lectores respondían con actitudes mucho más pesimistas y condenatorias. Por otro lado, las distintas partes de la discusión tenían mucho más en común de lo que parecía a primera vista. Todos, de hecho, consideraban la sociedad capitalista contemporánea como una poderosa fuente de envilecimiento moral e intelectual, y celebraban productos culturales tradicionales (como la música clásica), o más recientes (como el cine neorrealista) como criterios para medir las virtudes culturales de los jóvenes. De manera igualmente monolítica, todos coincidían en señalar la música popular moderna (a la cual referían con el apelativo de «música ye-ye») como el principal exponente de la crisis moral y cultural de la Italia de mediados de la década del 60, que hacía de los más jóvenes sus víctimas principales.

\section{¿A dónde van los pelilargos?}

Este estado de cosas, sin embargo, mostró un cambio crucial muy poco tiempo después. A lo largo de 1966, de hecho, el debate entre los lectores de Vie Nuove retomó la intensidad de 1964, pero esta vez en términos mucho más matizados y complejos. Ya a principios de 1966, por ejemplo, la revista publicó dos cartas de lectores preguntando acerca de los Beatles y qué era ese fenómeno llamado «beatnik.» La respuesta del periodista era altamente informativa, lo cual no evitaba que hacia el final se permitiera una opinión, sugiriendo que los nuevos movimientos culturales merecían una «equilibrada comprensión» que permitiera separar los

${ }^{41}$ «Ancora sulle giovani generazioni,» Vie Nuove, n. ${ }^{\circ}$ 38, 17 de Septiembre 1964. 
elementos válidos de aquellos que le parecían productos de las modas y las industrias culturales. Al fin y al cabo, concluía el periodista, «estamos frente a movimientos de rebelión contra los comportamientos rígidos, extrañados y conformistas de las sociedades industriales anti-democráticas.» El pelo largo en los jóvenes, de hecho, le parecía entonces como el ejemplo perfecto de esta clase de rebeldía. ${ }^{42}$

Las reflexiones del periodista evidenciaban una actitud nueva de la revista, en donde elementos intrínsecos y rupturistas de la cultura juvenil empezaban a valorarse más positivamente. Este cambio de actitud, de alguna manera, ya se podía detectar en algunas conclusiones del año anterior. Un ejemplo claro de esto es la cobertura de la gira de 1965 de los Beatles por Italia, en donde la corresponsal de Vie Nuove mostraba una actitud matizada frente al cuarteto. ${ }^{43}$ La cronista presentaba a los cuatro de Liverpool como jóvenes procedentes de hogares obreros que en su itinerario cultural habían pasado por la bohemia anticonformista para luego ser absorbidos por la industria musical. Esto último, según su análisis, se podía leer en el contraste entre su pelo largo, que ella no censuraba, y los trajes formales que usaban como si fueran uniformes, algo que le parecía impostado. El artículo cerraba entonces en abierta polémica contra los excesos de intelectualización o los alarmismos con respecto al fenómeno de los Beatles, concluyendo que si bien los Beatles eran un producto comercial, detrás del producto había cuatro muchachos simpáticos, desinhibidos, espontáneos y divertidos, cantándole a las «ganas de vivir.» Nada demasiado malo podía salir de ahí. ${ }^{44}$

Poco a poco, entonces, comenzó a surgir dentro de la revista una corriente de opinión menos condenatoria de la cultura juvenil, y más sensible a detectar aportes válidos en muchas de las prácticas y costumbres

42 Gianni Toti, «Beatles e beatniks: ossia, poessia e rivolta,»Vie Nuove, n. ${ }^{\circ}$ 4, 27 de Enero de 1966.

${ }^{43}$ La gira de los Beatles por Italia incluyó las ciudades de Roma, Milan, y Génova. Para una referencia al impacto en la prensa del momento, Giachetti, Anni sessanta, pp. 93-100.

44 Annamaria Rodari, «I Beatles in Italia. In flagrante delirio,»Vie Nuove, n. ${ }^{\circ}$ 26, 7 de Julio de 1965. Este análisis no dejaba de marcar un distanciamiento de algunas posiciones más enconadas, que sugerían paralelismos entre la fascinación por los Beatles y el fanatismo juvenil por el fascismo durante la entre-guerra. Véase una breve discusión al respecto en la mesa redonda sobre la juventud organizada por Vie Nuove: «Sesso e Societá. I giovani, l'amore e i tabú,»Vie Nuove, n. ${ }^{\circ}$ 19, 12 de Mayo de 1966. Para más ejemplos de esta hostilidad, ver también Gundle, Between Hollywood and Moscow, pp. 111-112. 
distintivas de la nueva generación. Esto fue acompañado por un creciente enfrentamiento dentro del mundo de Vie Nuove. De hecho a lo largo de 1966 el correo de lectores de la revista fue el espacio de contienda de diversas posturas sobre el significado del movimiento beat, el pelo largo y los nuevos estilos musicales ${ }^{45}$ En contraste con la homogeneidad de criterios de los debates anteriores, en este caso las diferencias fueron ciertamente profundas. En un extremo se pueden encontrar casos de abierto entusiasmo con las nuevas costumbres, manifestándose con un desenfado impensable apenas unos meses antes. Un joven de 19 años de Génova, por ejemplo, refería en una carta que cuando iba a bailar o a escuchar grupos modernos se sentía tan liberado de la timidez y la pesantez habitual que «a la salida me siento libre, me siento otro, no estoy más condicionado por la sociedad, es como si hubiera ido al psicoanalista.» Tal cual concluía el entusiasta bailarín, «el fenómeno beat, los cabellos largos, (...) etc, son fenómenos que (...) deben ser considerados como un camino natural hacia la vida moderna, hacia un tipo de relaciones más humano,

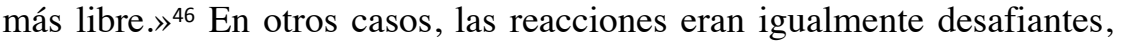
pero apoyadas en la instrumentalidad política. Así entonces un participante de 36 años de Roma observaba que las letras de las canciones de los nuevos grupos juveniles, así como las manifestaciones contra el racismo y la guerra en Estados Unidos, mostraban que en las nuevas culturas juveniles había un potencial político asombroso, y que las viejas generaciones lo estaban desaprovechando por actuar con prejuicios. El lector concluía entonces que «me parece absurdo que la carga objetivamente revolucionaria de los jóvenes no encuentre modo de ser organizada por los organismos juveniles de los partidos democráticos,» y que eran las organizaciones las que debían aprender a adecuarse a los jóvenes, y no viceversa. ${ }^{47}$ Finalmente, no faltaban quienes participaban en el debate haciendo un llamado

45 El debate parece haberse iniciado hacia Mayo de 1966, cuando dos lectores escribieron atacando la moda del pelo largo entre los jóvenes: véase «Ancora qualche parola sui capelloni,»Vie Nuove, n. ${ }^{\circ}$ 20, 19 de Mayo de 1966, y la inmediata respuesta: «Un dibattito su Beatles e shake,»Vie Nuove, n. ${ }^{\circ} 21,26$ de Mayo de 1966. En total, cartas participando del debate se pueden encontrar en Vie Nuove, 20, 21, 25, 27, 28, 30, 32, 33, 35, 37, 38, 39, 40, 41 abarcando entonces desde Mayo hasta Octubre de ese año. Véase también la encuesta entre colaboradores de Vie Nuove, en la que cada uno escribe su opinión sobre los jóvenes y el pelo largo: «Capelloni alla sbarra. Cosí li vedono i nostri collaboratori»Vie Nuove, n. ${ }^{\circ}$ 49, 8 de Diciembre de 1966.

46 «Un dibattito su shake e beat,» Vie Nuove, n. ${ }^{\circ}$ 25, 23 de Junio de 1966.

47 «Ancora su 'shake' e 'beatnik'» Vie Nuove, n. . 33, 18 de Agosto de 1966. 
a la tolerancia. Un comerciante de 40 años de la ciudad de Ancona, por ejemplo, se quejaba de la superficialidad de gran parte de las críticas a los jóvenes de pelo largo, y creía que el eje de la discusión tenía que centrarse en la tolerancia por formas de vida diferentes a las aceptadas por la mayoría. Recordando su experiencia de partisano en 1944, no dejaba de observar elocuentemente que los fascistas llevaban el pelo corto, y sugería una comparación entre la discriminación contra los pelilargos y la censura por la moda beat y las minifaldas con el racismo de la época del régimen. ${ }^{48}$

Entusiasmo, racionalidad política, y tolerancia eran entonces posiciones significativas e importantes a favor o en defensa de las nuevas culturas juveniles. Si bien prominentes y desenfadadas, estas voces no monopolizaban la representatividad de todos los lectores. En el otro extremo del espectro del mundo de Vie Nuove, de hecho, se encontraban posturas de adultos y jóvenes claramente desfavorables, aunque con diversos grados de hostilidad. Por un lado, por ejemplo, estaba la postura que se podría definir como «realismo escéptico»: condenar una determinada expresión cultural refiriendo al inmenso negocio detrás de él. Tal cual observaba un lector, «¿pero quiénes son estos Beatles? ¿Jóvenes que ganan fortunas por romper los tímpanos de los gentlemen de vieja estampa? En realidad (y no solo en el caso de los Beatles) se trata de todo un movimiento publicitario para engañar a los inocentes jovenzuelos y hacer dinero... $\gg^{49}$ Esta idea se complementaba, a veces en una misma carta, con la noción de que en la sociedad capitalista la alienación de la juventud generaba falsas respuestas al descontento social. El resultado entonces era que el movimiento beat, el pelo largo y todas las actitudes de supuesto anti-conformismo y rebelión eran en realidad una manera de lucrar con la sana rebeldía de la juventud. De acuerdo al lector, este análisis podía llevar a la simple indiferencia o, más comúnmente, a identificar a la cultura juvenil como a un enemigo. Tal cual concluía un lector realizando este análisis, «todos estos fenómenos exhibicionistas que nos circundan (...) no hacen otra cosa que alejar a los jóvenes de la política, desviando sus intereses hacia otros sectores de la vida social.» Era por eso que de acuerdo a este lector, «la prensa democrática debería batirse todavía más de cuanto lo hace hasta ahora para tratar de abatir todos los falsos mitos que nos circundan..${ }^{50}$ 1966.

48 «Sui beats con violenza e con equilibrio,» Vie Nuove n. ${ }^{\circ} 37,15$ de Septiembre de

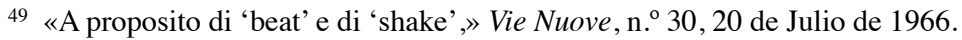

50 «Ancora sui capeloni,»Vie Nuove, n. ${ }^{\circ}$ 36, 8 de Septiembre de 1966. 
Finalmente, ya en el máximo de hostilidad, se encontraban las posturas abiertamente moralistas y que atacaban a las culturas juveniles con un tono de intensa indignación. Así entonces, un estudiante romano de 25 años se preguntaba:

¿creen los beats que se puede mejorar el mundo con las guitarras, gritando canciones sin sentido que no hacen otra cosa que arrojar a la vergüenza a los estúpidos nenes de papá y las histéricas en minifalda vistiendo excéntricamente y viviendo en la suciedad y la inmoralidad? (...) Y las mujeres, ¿creen en mejorar el mundo mostrando las piernas y llevando eso que de más bello hay en el amor de una mujer al nivel del desprejuicio y la inmoralidad(...)? ${ }^{51}$

No es fácil detectar hasta qué punto este último tipo de vocabulario y estas percepciones estaban extendidas entre los comunistas italianos. En principio no habría que dejar de mencionar que exabruptos como éste eran fuertemente criticados por distintos lectores, incluso aquellos más escépticos con respecto a la cultura juvenil. ${ }^{52} \mathrm{Sin}$ embargo, este género de declaraciones parecen ser representativos de una actitud muy conservadora en algunos militantes o simpatizantes del PCI, y que tiene que ver con una excesiva sensibilidad por no romper ciertos parámetros de respetabilidad. Un caso paradigmático de esta actitud era la carta justamente titulada «una madre contra los beats» escrita por una señora de Bolonia que se vanagloriaba de que «mi hijo es un joven moderno, equilibrado, normal como tantos otros. Sobre todo, no es un beat y no es un caso patológico.» Enojada porque se le daba demasiada importancia a los beats, la señora reproducía ideas similares a la del estudiante romano. Sin embargo, todavía más significativo era la forma en que cerraba su carta,

El partido comunista, ¿tiene necesidad del número o de la calidad? Si tiene necesidad del número entonces que acepte [a los beats]. Pero la consecuencia será de retroceder dieciséis o dieciocho años, a los tiempos en los que, cuando se hablaba de un comunista la gente imaginaba un pobretón andrajoso, y se maravillaba cuando veía personas distintas, finas y elegantes, profesar ideas comunistas. ${ }^{53}$

51 «Sui beats con violenza e con equilibrio»Vie Nuove, n. ${ }^{\circ} 37$, cit.

52 Ver por ejemplo, «Battaglia per i beats... secondo round,»Vie Nuove, n. ${ }^{\circ}$ 39, 29 de Septiembre de 1966.

53 «Una mamma contro i beats»Vie Nuove, n. ${ }^{\circ}$ 40, 6 de Octubre de 1966. 
Tal vez más que en muchas otras intervenciones, en la última carta citada se puede ver uno de los principales problemas de la relación entre los comunistas y las culturas juveniles de la década de 1960. Luego de años de pelea por la aceptación social y la respetabilidad, ahora muchos viejos comunistas veían cómo una nueva camada de jóvenes reclamaban precisamente todo lo contrario: romper con las pautas de decoro y aceptabilidad proclamadas por la sociedad oficial. Esto no era siempre fácil de procesar, tal cual demuestran las reacciones negativas sobre las expresiones de la cultura juvenil. Sin embargo, más allá de estas posturas duras y represivas, convivieron también, en el seno de militantes, intelectuales y dirigentes vinculados al comunismo, un amplio espectro de posturas. Y este es tal vez uno de los aspectos más dignos de mención a la hora de resumir la experiencia politico-cultural subyacente al PCI: el de constituir un espacio ideológico en donde posturas más que heterogéneas y hasta antagónicas supieron convivir, discutir, y polemizar.

\section{Conclusiones: Modernización, Juventud y Política}

No debería sorprendernos que las evaluaciones sobre la cultura juvenil hayan sido tan heterogéneas y contradictorias. En una época de abruptos cambios y obstinadas continuidades los balances sobre las nuevas tendencias culturales se enfrentaban con el problema de aferrar un proceso esquivo y transicional, cuyas verdaderas dimensiones solo podían llegar a vislumbrarse con la perspectiva del largo plazo. Mientras que la década comienza con observaciones acerca de la indiferencia cívica y política de los jóvenes, o con muestras de pánico moral con respecto a sus actitudes criminales y anti-sociales, hacia el fin de la misma los retratos son muy diferentes, y el modelo de la radicalización política estudiantil pasa a monopolizar la representaciones que se elaboran sobre la juventud. Las miradas y percepciones elaboradas por la comunidad de lectores de Vie Nuove a mediados de los 60s se tornan interesantes precisamente por su indefinición. Se trataba de aferrar el sentido de un cambio cultural significativo en el momento preciso en que se estaba desarrollando, y cuando las miradas que juzgaban y evaluaban dichas transformaciones estaban también siendo parte de esos cambios.

De hecho, tratar de encontrar las continuidades y rupturas entre los jóvenes consumistas y supuestamente despolitizados de los ' $50 \mathrm{~s}$, y los movilizados de los ' 60 s tardíos es un desafío para los historiadores más allá 
del caso italiano. Así por ejemplo, Eric Hobsbawn considera que el consumismo y la contracultura radicalizada tardo-sesentista tienen más en común de lo que parecería a primera vista, puesto que lo que las unifica es el principio de defender y fomentar la «autonomía ilimitada del deseo individual.» ${ }^{54}$ Para Tony Judt, en cambio, los jóvenes de los '50s y tempranos ' 60 s fueron una suerte de rebeldes a pesar de sí mismos, puesto que lo que buscaban no era cambiar el mundo sino divertirse e identificarse de maneras distintas a las consagradas por lo adultos - lo cual despertaba todo tipo de temores y alarmismos..$^{55}$ Esto explicaría también por qué la esfera del consumo juvenil y sus productos distintivos - vestimentas, música, entretenimientos - se vincularía con formas contraculturales y políticas de los ' 60 s tardíos. La continuidad no estaría tanto en los contenidos sino en el «estilo» irreverente y, a su manera, rupturista, desenfadado y desinhibido de los productos culturales juveniles de la época. Como concluye Judt tomando el ejemplo de la cultura pop de los ' 60 s: «La música, por decirlo de alguna manera, protestaba por vos.» ${ }^{56}$

Historiadores de la Italia de posguerra han estado también atentos a detectar la manera en que conviven en su seno la asimilación del mundo del entretenimiento moderno y «americanizado» con formas implícitas de contestación política y cultural. Así entonces, Alessandro Portelli, en su estudio sobre la recepción del rock-and-roll en Italia, enfatiza su centralidad para la conformación de la cultura juvenil en la península - que encontró en los cantantes y ritmos llegados de los Estados Unidos un estilo dirigido por primera vez exclusivamente a adolescentes. Si bien no niega el carácter conservador del rock de los ' 50 s, Portelli no deja de recordar el espanto que provocaba en padres, enseñantes y autoridades eclesiásticas. Para quienes lo consumían y bailaban en Italia, el rock era entonces una transgresión: «invitando [incoraggaindo] a los muchachos a pensar que estaban violando reglas y costumbres, les enseñó que la transgresión era una cosa justa, incluso si luego en realidad no transgredían nada de concretamente importante.» De ahí la fórmula tajante con la que Portelli concluye, «de los teenagers de los años ' 50 s salieron los movimientos estudiantiles del decenio siguiente. $\rangle^{57}$

\footnotetext{
${ }^{54}$ Hobsbawm, The Age of Extremes, pp. 333-334.

55 Judt, Postwar, p. 349.

56 Idem, p. 396.

57 Portelli, «L'orsachiotto,» p. 146.
} 
En uno de los estudios más prolongados y detallados sobre la cultura juvenil en Italia, Piccone Stella hace una observación que ilumina lo contradictorio y «ambiguo» de la misma. Haciendo referencia a las actitudes, espacios de sociabilidad, y formas de agruparse de adolescentes de fines de los '50s y principios de los ' 60 s, concluye que éstos implicaban una inclinación a la «desadaptación y al malestar.» Sin embargo, estos rasgos no se dirigían como una protesta contra la sociedad de consumo, sino todo lo contrario: la insatisfacción con las costumbres y actitudes tradicionales entre los jóvenes implicaban una celebración del boom económico. En su promesas de abundancia, culto de la novedad, expansión de roles sociales y espacios de sociabilidad, hedonismo, y ausencia de moralismo, los y las adolescentes italianos de los ' $50 \mathrm{~s}$ vieron una posibilidad de liberación. ${ }^{58}$ Sin embargo, y como los autores anteriores, el marcar esta diferencia no quita que para Piccone Stella la cultura juvenil que se conforma en los '50s constituye un unidad que se expande hasta los rebeldes y politizados movimientos de fines de los '60s. De hecho, para ella lo que comienza en los '50s son dos cosas: el conformarse de una cultura juvenil autónoma, en ruptura con el mundo de los adultos; y el hecho de que esta cultura implique variadas formas de inconformismo, antagonismo, e inclinación a la transgresión. ${ }^{59}$

Mientras que las características complejas y contradictorias de la cultura juvenil de posguerra llamaron la atención a historiadores actuales, fueron mucho más esquivas e indescifrables para sus contemporáneos. En el caso de la comunidad de lectores de Vie Nuove, podemos de hecho rescatar el desconcierto, las preocupaciones, incertidumbres y ambivalencia de jóvenes y adultos ligados al comunismo italiano. Para ellos, la cultura juvenil, el surgir de actitudes propias y contrastantes con el mundo de los adultos por parte de muchachos y muchachas italianos, se les presentaba como un entramado de obstáculos y posibilidades. De esta forma, quienes construyeron su modelo de juventud politizada de acuerdo a pautas (reales o romantizadas) surgidas en el momento de la Resistencia, vieron con preocupación la creciente diferenciación generacional de una juventud que, según ellos, depositaba sus energías en otras actividades. De la misma manera, quienes habían conocido las presiones durante la inmediata posguerra para marginar y demonizar al comunismo, valoraron la

\footnotetext{
58 Piccone Stella, Prima generazione, pp. 10-13.

59 Piccone Stella, «Rebels without a Cause», p. 170; y «All'origine della cultura giovanile,» p. 149.
} 
conquista de la respetabilidad como un triunfo cultural importantísimo. Es claro que en esta perspectiva, la modas juveniles provocadores e insinuantes de los '60s aparecían como un incomprensible deseo de auto-exclusión. Sin embargo, estas voces, importantes como fueron, no monopolizaron la percepción de los comunistas de Vie Nuove. La búsqueda por tratar de entender y valorar las prácticas y formas novedosas de entretenimiento y acción juvenil tuvieron también su lugar en la revista. Se trataba de hecho de una usina de inconformismo que, tal cual lo entendieron muchos, debían buscar capitalizar en su favor, tolerar, o incluso abandonarse a sus ritmos y estilos con genuino entusiasmo.

El hecho de que en el seno de una comunidad ideológica como lo eran los lectores de Vie Nuove haya existido tal heterogeneidad de perspectivas nos parece entonces el rasgo más digno de resaltar. En primer lugar porque nos remite a la diversidad dentro del PCI, que en tanto partido de masas, fue capaz de englobar bajo un mismo signo político diversas sensibilidades e incluso formas antagónicas de valorar lo personal. Se puede percibir entonces cómo un estudiante, un joven obrero, una ama de casa, o un profesor jubilado votaban de la misma manera a pesar de tener percepciones muy distintas sobre temas culturales cruciales. En segundo lugar porque aporta para entender el desconcierto que provocó el aparecer de una cultura propiamente juvenil en la Italia de los ' 60 s. Lo que sorprende al leer las cambiantes y contrastantes reacciones de lectores y editores de Vie Nuove es la increíble fluidez de significados en un contexto social cambiante. De esta manera, prácticas específicas recibían una interpretación muy distinta casi sin solución de continuidad. Episodios de vandalismo y resistencia a la autoridad entre jóvenes abonaban a las preocupaciones acerca de una «juventud perdida» como efecto de los males capitalistas. Pero cuando manos jóvenes arrojaban piedras a policías en una marcha anti-fascista, la energía rebelde juvenil se volvía un ejemplo de heroísmo cívico. De la misma manera, la brecha generacional era negada en un comienzo, para acentuar la armonía y la continuidad entre generaciones. Sin embargo, ya hacia mediados de la década del '60, las prácticas distintivas de los jóvenes pasaban a contener un potencial innovador y rebelde digno de apreciación. Finalmente, incluso el americanismo recibía interpretaciones ambiguas. El proliferar de estilos musicales, vestimentas y formas de entretenimiento influenciados por la industria cultural norteamericana levantaban más que sospechas en el marco de los comunistas de la época de la guerra fría. Pero cuando esas influencias se 
revelaban como críticas del militarismo y el racismo norteamericano, de pronto se volvían aliados ideológicos.

La cultura juvenil italiana fue ciertamente un fenómeno complejo en una sociedad en donde los criterios mismos de evaluación estaban lejos de ser estables. A diferencia de historiadores posteriores, quienes buscaron entenderla en su momento la encontraron como una realidad fluida hacia destinos que no podían entrever claramente. Llegar a juicios tajantes o formulas definitivas fue ciertamente una tarea más que esquiva para los comunistas de Vie Nuove que, con distintos grados de alarmismo, temor, dogmatismo, curiosidad, tolerancia, o entusiasmo, trataron de decodificar y descifrar las líneas directrices de un mundo de valores, prácticas y significados novedosos. Podríamos preguntarnos hasta qué punto las percepciones de la comunidad de Vie Nuove son originales o radicalmente distintas con respecto a la de otras esferas ideológicas o sociales. De la misma manera, no deberíamos dejar de concluir que más allá de las diversas reacciones por parte de Vie Nuove, existió una actitud que ciertamente unificó y dominó los debates. Frente a una realidad elusiva que suscitaba todo tipo de emociones y aprehensiones, la iniciativa más constante fue la de abrir el debate entre voces disonantes. Esto ciertamente dejó un material valioso para el historiador actual, así como un testimonio de la unidad en la diversidad que constituyó al PCI en una de las experiencias políticas más llamativas de la Europa de posguerra. 